\title{
Identification of a potential exosomal biomarker in spinocerebellar ataxia Type 3/Machado-Joseph disease
}

\author{
Xiaocan Hou ${ }^{1}$, Xuan Gong ${ }^{2}$, Longbo Zhang ${ }^{2}$, Tianjiao Li ${ }^{1}$, Hongyu Yuan ${ }^{1}$, Yue Xie ${ }^{1}$, Yun \\ Peng $^{1}$, Rong Qiu ${ }^{3}$, Kun Xia ${ }^{4}$, Beisha Tang ${ }^{1,4,5,6}$ \& Hong Jiang*,1,4,5,6 \\ ${ }^{1}$ Department of Neurology, Xiangya Hospital, Central South University, Changsha, Hunan 410008, PR China \\ ${ }^{2}$ Department of Neurosurgery, Xiangya Hospital, Central South University, Changsha, Hunan 410008, PR China \\ ${ }^{3}$ School of Information Science \& Engineering, Central South University, Changsha, Hunan 410008, PR China \\ ${ }^{4}$ Laboratory of Medical Genetics, Central South University, Changsha, Hunan 410008, PR China \\ ${ }^{5}$ Key Laboratory of Hunan Province in Neurodegenerative Disorders, Central South University, Changsha, Hunan 410008, PR China \\ ${ }^{6}$ National Clinical Research Center for Geriatrics Disorders, Xiangya Hospital, Central South University, Changsha, Hunan 410008, \\ PR China \\ *Author for correspondence: Tel.: +86 7318432 7216; Fax: +86 7318432 7332; jianghong73868@126.com
}

\begin{abstract}
Aim: To identify spinocerebellar ataxia Type 3 (SCA3)-related exosomal biomarkers and the underlying mechanisms. Materials \& methods: Exosomal RNAs from plasma and cerebrospinal fluid (CSF) were extracted from 24 SCA3 patients and 22 controls, respectively. Small RNA sequencing and quantitative PCR verification were performed. Gene ontology annotation and Kyoto Encyclopedia of Genes and Genomes pathway analyses of the results were carried out. Results: One novel miRNA is notably downregulated in plasma-derived exosomes, while upregulated in CSF-derived exosomes of SCA3 patients. Besides, it is successively upregulated in CSF-derived exosomes from Type 1, Type 2 and Type 3 groups. The downstream target genes were enriched in protein processing in endoplasmic reticulum and axon guidance. Conclusion: One exosomal biomarker was identified in SCA3, and this is the first time to report an exosomal miRNA as a biomarker in SCA3 internationally.
\end{abstract}

First draft submitted: 29 March 2019; Accepted for publication: 9 May 2019; Published online: 30 May 2019

Keywords: age at onset $\bullet$ axon guidance $\bullet$ biomarker $\bullet$ clinical classification $\bullet$ endoplasmic reticulum $\bullet$ exosomes $\bullet$ miRNAs $\bullet$ pathway $\bullet$ SCA3/MJD • subtype

Spinocerebellar ataxia Type 3, also known as Machado-Joseph disease (SCA3/MJD), is a group of highly heterogeneous neurodegenerative disorders with autosomal dominant inheritance, and usually appears as progressive ataxia and spasticity [1]. Some scholars have classified SCA3/MJD into the following subtypes: Type 1 begins early in life normally before age 20 , which may progress more quickly with prominent pyramidal signs (rigidity and spasticity) and extrapyramidal features (bradykinesia and dystonia) as well as ataxia; Type 2 has a moderate age of onset (20-50 years of age) with cerebellar ataxia, progressive external ophthalmoplegia and pyramidal signs; Type 3 has a later onset (40-75 years of age) and appears as peripheral signs such as motor neuropathy and muscle atrophy together with ataxia; Type 4 is characterized by parkinsonism associated with other core clinical features; and afterward, a Type 5 SCA3/MJD was recently proposed for pure spastic paraplegia [2]. Among these five subtypes of SCA3/MJD, Type 2 is the most frequent, followed by Type 3, Type 1 and then the rare Types of 4 and 5 [3].

SCA3/MJD is one of nine polyglutamine diseases (polyQ diseases), which is characterized by polyglutamine encoding CAG sequences. Healthy individuals have 12-40 CAG repeats, which is expanded to 51-86 repeats in SCA3/MJD patients [4-6]. ATXN3, the gene causatively associated with SCA3/MJD, is located on chromosome $14[7,8]$ and encodes a poly-Q-containing protein named ataxin-3 [7]. Ataxin-3 is a deubiquitinating enzyme present in the cytoplasm and nucleus that appears to function in cellular pathways regulating protein homeostasis as well as transcription [9-14]. Expansion of the polyglutamine domain destabilizes the mutant protein, leading to increased misfolding, altered interactions between proteins and neuronal toxicity and death. A feature of polyglutamine 
diseases is the formation of neuronal inclusions, which consist of misfolded disease proteins, ubiquitin, molecular chaperones, proteasomes and amounts of other proteins, generally in the nucleus [15-17]. Studies with cell [18-20] and transgenic mice [21-23] models showed that pathogenic ataxin-3 was more toxic when in the nucleus than in the cytoplasm. However, the molecular biomarkers and their accurate mechanism remain to be elucidated in SCA3/MJD.

Exosomes are tiny vesicles $(40-150 \mathrm{~nm})$ containing RNAs, DNAs and proteins that secreted by all living cells, which could be detected in various body fluids. Once attached to a target cell, exosomes can be internalized by the target cell's membrane to deliver their content, thereby modifying the physiological state of the recipient cell [24].

Although genetic diagnosis of SCA3/MJD is the gold standard for diagnosis, it cannot be used to estimate other aspects such as clinical clarification, the disease duration or the severity of ataxia [25]. In the past few years, there were only a few studies about the functional roles of exosomes in neurodegenerative diseases. First, biomarker research: Saman et al. [26] identified a variety of misfolded tau proteins in CSF-derived exosomes in Alzheimer's disease (AD); Mullins et al. [27] found that upregulated pSer312-IRS-1 and downregulated p-panTyr-IRS-1 in exosomes from $\mathrm{AD}$ patients were related to its brain atrophy degree, and the above two indicators were consistent with the regional expression of IRS-1. They both indicated that exosomes could be used as biomarkers for diagnosis and assessment for AD progression. Second, pathogenesis research: Stuendl et al. [28] found $\alpha$-synuclein in CSFderived exosomes in Parkinson's disease (PD) patients differs from that in dementia with Lewy bodies patients, and was associated with cognitive level. Through co-culturing normal mice neural stem cells with fibroblasts from huntington patients, Jeon et al. [29] found that exosomes could transfer mutant huntington proteins across species boundaries and then introduce relative performance of behavior and pathology. Third, potential therapeutic research: exosomes could pass through the blood-brain barrier easily due to their small size. With the addition of immune privilege character, exosomes are becoming a new promising therapeutic vector for neurodegenerative diseases increasingly [30]. Unfortunately, the roles of exosomes in SCAs have not been reported so far.

The idea that miRNAs (one group of noncoding RNAs with 21-25 nucleotides in length) secreted in exosomes can be functionally transferred to living cells, resulting in modulation of their target genes, has become one of the most actively explored hypotheses in recent years [24]. As exosomes isolated from extracellular fluids such as CSF and blood represent an enriched source of biomolecules, they are hypothesized to provide potential biomarkers for neurodegenerative diseases. The nervous system is a rich source of miRNAs [31], which may reflect clinical classification, the onset or stages of neurodegenerative disease [32]. While most studies utilize free miRNAs, so as our former research, to investigate the role of miR-25 in cell models of SCA3/MJD [33], exosomes provide a protective environment for miRNAs [34], which demonstrates the feasibility of using exosomal miRNA as an alternative biomarker screening tool for neurodegenerative diseases. However, profiling of exosomal miRNAs in SCA3/MJD patients' blood and CSF has never been illustrated.

In the present study, we explored the profile of exosomal miRNAs derived from the plasma and CSF of SCA3/MJD patients to identify the disease-related biomarker and investigate its potential molecular mechanism.

\section{Material \& methods}

\section{Recruitment of SCA3/MJD subjects \& SCA3/MJD-free controls}

Plasma and CSF samples were collected from the Department of Neurology and Neurosurgery in Xiangya Hospital, Central South University (China) from 8 August 2017 to 1 March 2018. All SCA3/MJD patients were under at least two experienced neurologists' clinical diagnosis in accordance with Harding diagnosis criteria and incorporated into this study based on the following criteria. First, the ages range from 18 to 75 years old; second, without other accompanying diseases such as hypertension, diabetes, etc. Third, without fever or traumatic brain injury within 1 month; and fourth, without intaking disease-related drug in the past 6 months. Besides, the neurological analyzing results of the subjects were settled using the mean calculated value of that obtained from these two neurologists. Only the first three relatively common subtypes of type 1, 2, 3 SCA3/MJD diseases were recruited and assigned to the corresponding groups on account of their age and clinical manifestations. Referred to SCA3/MJD-free controls, plasma samples were recruited from healthy volunteers and CSF samples were recruited from aneurysm or migraine patients with chronic headache without intracranial hypertension, whose indexes of routine biochemical and cytology in CSF were basically normal. The SCA3-free controls had a similar range of age and life conditions with SCA3/MJD patients. Both SCA3/MJD patients and SCA3/MJD-free controls had signed the informed consent. All samples were obtained in accordance with the ethical standards in the Declaration of Helsinki and the research was approved by Medical Ethics Committee of Xiangya Hospital, Central South University, China. 
As for plasma samples, plasma-derived exosomal RNAs from 12 SCA3/MJD patients (three cases of Type 1, six cases of Type 2 and three cases of Type 3), and 12 healthy controls with matched age and gender were extracted for small RNA sequencing. In addition, plasma-derived exosomal RNAs from 18 other patients (one case of Type 1 and 17 cases of Type 2) and 16 controls were extracted as the validation cohort for further real-time quantitative PCR (qPCR) verification. With respect to CSF samples, CSF-derived exosomal RNAs from 12 SCA3/MJD patients (three cases of Type 1, six cases of Type 2 and three cases of Type 3) and ten controls with matched age and gender were isolated for small RNA sequencing.

\section{Exosomes/exosomal RNA isolation \& identification}

About $4 \mathrm{ml}$ plasma or $15 \mathrm{ml}$ CSF was applied to separate exosomes and extract exosomal RNAs, respectively, according to the protocols of exoEasy Kit and exoRNeasy Serum/Plasma Kit (Qiagen, Hilden, Germany). The exosomes were identified by nanoparticle tracking (NTA) under a ZetaView Nanoparticles Trace Analyzer (Particle Metrix, Munich, Germany), a transmission electron microscope (Tecnai G2 Spirit, FEI, Hillsboro, Oregon) and by detection of exosomal marker (CD9) using western blot with antibodies purchased from Santa Cruz Biotechnology (CA, USA) [35,36]. The RNA quality testing of isolated exosomal RNAs was carried out using Agilent 2100 (CA, USA).

\section{Small RNA sequencing}

Small RNA sequencing was carried out to discover the profile of exosomal miRNAs derived from plasma and CSF samples of SCA3/MJD patients and SCA3/MJD-free controls. First, following RNAs quality control after exosomal RNA extraction, exosomal RNAs were sequenced using BGISEQ-500 technology (BGI, Shenzhen, Guangdong, China) with 20M reads. The raw data received a subsequent bioinformatics analysis (Supplementary File 1). Second, data filtering: the data were processed using the following steps: remove low quality tags; remove tags with $5^{\prime}$ primer contaminants; remove tags without $3^{\prime}$ primer; remove tags without insertion; remove tags with poly A; remove tags shorter than $18 \mathrm{nt}$; summarize the length distribution of the clean tags. Third, reads mapping: the software of Bowtie [37] was used to map clean reads to the reference genome and to other small RNA databases. Fourth, miRNAs prediction: miRDeep2 [38] was employed to predict novel miRNAs by exploring the characteristic hairpin structure of miRNA precursors. Fifth, miRNA expression: the levels of miRNAs expression were calculated by using Transcripts Per Kilobase Million, which was able to eliminate the influence of sequencing discrepancy on the calculation of small RNA expression [39]. Sixth, target prediction: miRanda [40] and TargetScan [39] were applied to predict target genes of miRNAs. Seventh, screening differentially expressed miRNAs: small RNA sequencing could be modeled as a random sampling process, each read was sampled independently and uniformly from every possible nucleotide in the samples [41]. Under this assumption, the number of reads coming from a transcript isoform followed a binomial distribution and could be approximated by a Poisson distribution. DEG seq (differentially expressed gene sequencing) analysis [42] using MA-plot, which was a statistical analysis tool having been widely used to detect and visualize intensity-dependent ratio of microarray data, was further carried out for screening of differentially expressed genes [43]: we defined $M=\log _{2} C_{1}-\log _{2} C_{2}$ and $A=\left(\log _{2} C_{1}+\log _{2} C_{2}\right) / 2$, where $C_{1}$ and $C_{2}$ were defined by $C_{i}$ approximately binomial $\left(n_{i}, p_{i} ; i=1,2, n_{i}\right.$ denoted the total number of mapped reads and pi denoted the probability of a read coming from that gene) to denote the counts of reads mapped to a specific gene obtained from two samples. Under the random sampling assumption, the conditional distribution of $\mathrm{M}$ given that $\mathrm{A}=\mathrm{a}$ ( $\mathrm{a}$ is an observation of $\mathrm{A}$ ) was proved to follow an approximate normal distribution. For each gene on the MA-plot, we did the hypothesis test of $\mathrm{H}_{0}: \mathrm{p} 1=\mathrm{p} 2$ versus $\mathrm{H}_{1}: \mathrm{p} 1 \neq \mathrm{p} 2$. Then a $\mathrm{p}$-value was assigned based on the conditional normal distribution. The p-values calculated for each gene was adjusted to Q-values for multiple testing corrections by two alternative strategies [44]. To improve the accuracy of DEGs result, we defined the genes whose fold change of reading number $\geq 2$ and Q-value $\leq 0.001$ as DEGs [44,45]. To improve the accuracy of these results, a miRNA was defied as a differentially expressed miRNA when reads number fold change $\geq 2$ and Q-value $\leq 0.001$. Eighth, hierarchical clustering analysis: hierarchical clustering for differentially expressed miRNAs was performed using heatmap, a function of R program. Ninth, gene ontology (GO) enrichment analysis: GO enrichment analysis was used to recognize the main biological functions that differentially expressed miRNA targets exercise: to perform this analysis, all genes were mapped initially to GO-terms in the database (www.geneontology.org/) to calculate the gene numbers for every term. 
The hypergeometric test basing on 'GO:: Term Finder' (www.yeastgenome.org/help/analyze/go-term-finder) was then used to find significantly enriched GO-terms in the input gene list. The algorithm of $P=1-\sum_{i=0}^{m-1} \frac{\left(\begin{array}{c}\mathrm{i} \\ \mathrm{i}\end{array}\right)\left(\begin{array}{c}\mathrm{N}-\mathrm{M}) \\ \mathrm{n}-\mathrm{i}\end{array}\right.}{\left(\begin{array}{c}\mathrm{N}) \\ \mathrm{n}\end{array}\right.}$ ( $\mathrm{N}$ represented the number of all genes with GO annotation, $\mathrm{n}$ represented the number of differentially expressed miRNAs' target genes in N, M represented the number of all genes that are annotated to a specific GO term and $\mathrm{m}$ represented the number of differentially expressed miRNAs' target genes in M) was used to perform this analysis. After that, the p-value was corrected by the Bonferroni method [46], where a corrected $\mathrm{p}$-value $\leq 0.05$ was taken as a threshold GO terms fulfilling this condition were defined as significantly enriched GO terms. Tenth, pathway enrichment analysis: pathway-based analysis helped to further understand the biological functions of differentially expressed miRNA-targeting genes. Kyoto Encyclopedia of Genes and Genomes (KEGG) [47] (the major public pathway-related database) was used to identify significantly enriched metabolic pathways or signal transduction pathways in differentially expressed miRNA-targeting genes comparing with the whole genome background: The calculating formula was the same as that in GO analysis, in which $\mathrm{N}$ was the number of all genes with KEGG annotation, $\mathrm{n}$ was the number of differentially expressed miRNAs' target genes in $\mathrm{N}$, M was the number of all genes that are annotated to a specific pathway and $\mathrm{m}$ was the number of differentially expressed miRNAs' target genes in $\mathrm{M}$. The $\mathrm{p}$-value was also corrected by the Bonferroni method and a corrected $\mathrm{p}$-value $\leq 0.05$ was taken as a threshold. KEGG terms fulfilling this condition were defined as significantly enriched KEGG terms.

\section{Verification of miRNAs by qPCR}

Further verification of the differential expression of the miRNAs detected above was carried out among plasmaderived exosomal RNAs from 18 other SCA3/MJD patients and 16 SCA3-free controls using qPCR method. Among the differentially expressed miRNAs, those had differential expression trends in more comparing groups and with relatively higher fold change were chosen for the verification preferentially. Combining with our study, we chose 27 miRNAs for further qPCR verification among the plasma-derived exosomal RNAs in the validation cohort. The specific PCR primers were designed and synthesized by TSING KE Biological Technology (Beijing, China). First, the reverse transcription was carried out using miScript II RT kit (Qiagen). The qPCR amplification for individual miRNA expression was carried out with $2 \times$ T5 Fast qPCR Mix (SYBR Green I) on a QuantStudio ${ }^{\text {TM }} 5$ Real-Time PCR System (Thermo Fisher Scientific, MA, USA) using 384-well plates (the primers of the miRNAs for verification are provided in Supplementary File 2). Cycle threshold $(\mathrm{Ct})$ values calculated as $\Delta \mathrm{Ct}=\mathrm{Ct}(\mathrm{miRNA})-\mathrm{Ct}$ (U6) method were obtained for further data analysis.

\section{Statistical analysis}

Relative expression levels of miRNAs were evaluated by comparing $-\Delta \mathrm{Ct}$ values. Box plots were generated using the software of R Studio. The differences between two groups fitting the normal distribution were analyzed using Student's t-test. Depending on the frequency histogram of data distribution which did not fit the normal distribution completely, nonparametric test was chosen for statistical analysis using SPSS20.0 (IL, USA). All statistical tests in the study were two sided and statistical significance was set at $\mathrm{p}<0.05$. Besides, linear-regression, Random Forest and LASSO analyses were carried out for the correlation of miRNAs and clinical indexes such as age at onset, CAG repeats, SARA scales and disease duration.

\section{Results}

\section{Clinical \& genetical characters of SCA3/MJD patients \& SCA3/MJD-free controls}

Clinical and genetical information of the subjects are detailed in Table 1: Among all the SCA3/MJD patients, there were 20 male patients (55.56\%) and 16 female patients (44.44\%); among all the SCA3/MJD-free controls, there were 19 males (50\%) and 19 females (50\%). Through subtype classification, the ages of subtype one patients were $22.8 \pm 4.3$ years old, while the ages at onset were $17.0 \pm 1.4$ years old; the CAG repeats of which range among $77.4 \pm 2.5$ repeats. As for subtype two, the ages of patients were at $40.4 \pm 8.5$ years old, the ages at onset were at $32.9 \pm 8.3$ years old; while the CAG repeats fluctuated among $71.8 \pm 3.8$ repeats. The age of subtype three were at $56.5 \pm 4.7$ years old; the ages at onset were $47.8 \pm 6.8$ years old; the CAG repeats ranged at $62.5 \pm 5.7$ repeats. The clinical characteristics were also obvious in accordance with the classification criteria of the three subtypes, in other words, Type 1 manifests prominent pyramidal signs (rigidity and spasticity) and extrapyramidal features (bradykinesia and dystonia) combined with ataxia, Type 2 has progressive external ophthalmoplegia and pyramidal signs with ataxia. Type 3 appears as peripheral signs together with ataxia. 


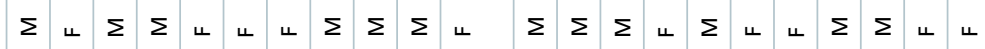

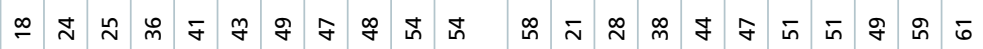

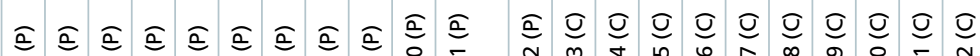

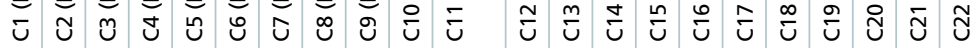

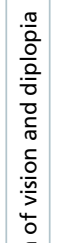

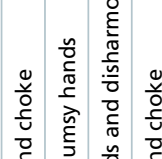

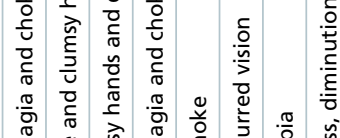

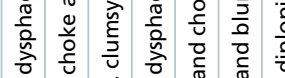

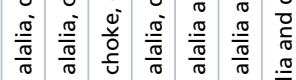

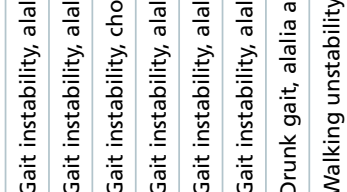
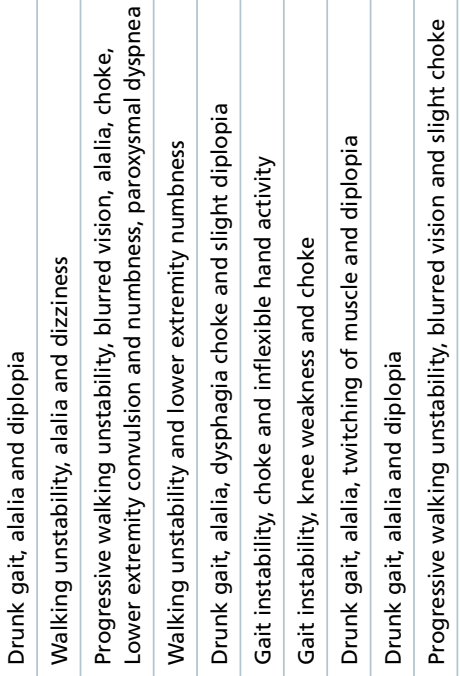

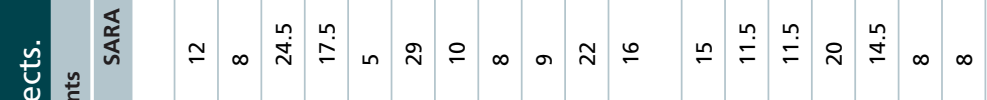
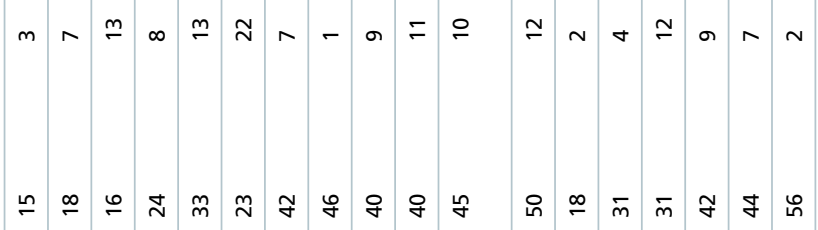


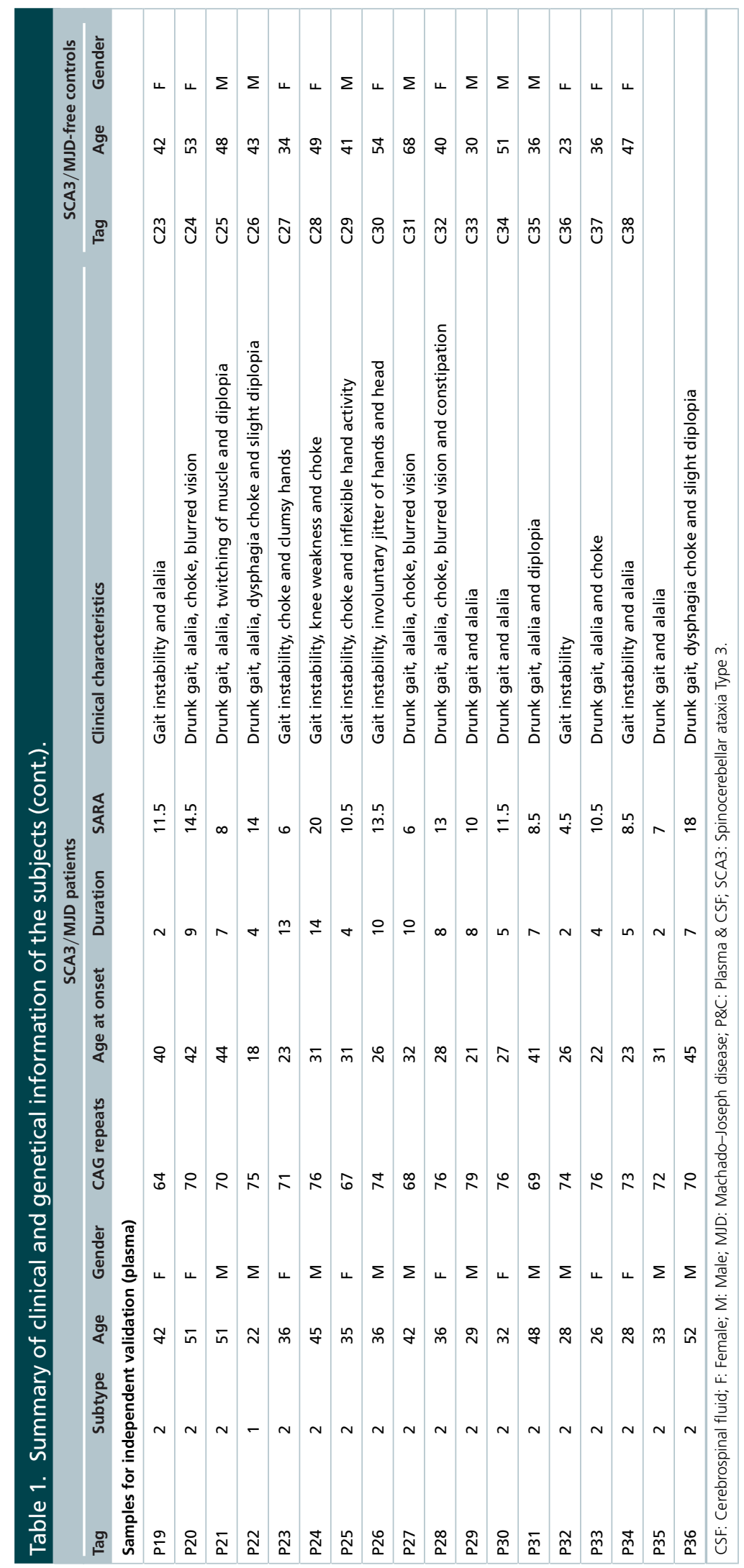




\section{Identification of isolated exosomes/exosomal RNAs}

Exosome numbers and scales shown in Figure 1 indicated that the particle size distribution was as follows: $10 \%$ of the particles were under $81.2 \mathrm{~nm}, 50 \%$ were under $135.4 \mathrm{~nm}$ and $90 \%$ were under $202.5 \mathrm{~nm}$. The exosomes were observed under the transmission electron microscope as round- or oval-like particles with uneven sizes, which distributed between $40-150 \mathrm{~nm}$ in diameter. Normally, they had complete membrane structures and encapsulated low density material inside. Besides, it was confirmed that there was an expression of exosome marker CD9 in the isolated particle proteins, which further identified the extracts as exosomes (Figure 1A-C).

\section{Differential expression of miRNAs in plasma-derived exosomes}

Through preliminary RNA sequencing analysis, 53 miRNAs (30 novel miRNAs and 23 known miRNAs) were found to be upregulated, 20 miRNAs (15 novel miRNAs and five known miRNAs) were found to be downregulated significantly ( $\mathrm{p}$-value $=0$, Q-value $=0$ ) in exosomes derived from SCA3/MJD patients' plasma, as being compared with that from SCA3/MJD-free controls (as shown in Table 2 and Figure 1D of hierarchical clustering analysis heatmap for details).

\section{Differential expression of miRNAs in CSF-derived exosomes}

As for CSF samples, 69 miRNAs (41 novel miRNAs and 28 known miRNAs) were detected to be upregulated, and 47 miRNAs (21 novel miRNAs and 26 known miRNAs) be downregulated significantly in exosomes derived from SCA3/MJD patients' CSF, as being compared with that from SCA3/MJD-free controls (p-value = 0, Q-value = 0, shown in Table 2 and Figure 1D of hierarchical clustering analysis heatmap).

\section{Comparison between exosomal RNAs derived from plasma \& CSF samples}

Based on a comparison of the differentially expressed miRNAs between plasma- and CSF-derived exosomes in SCA3/MJD subjects, the expression trend of 15 miRNAs reached consensus between them, while ten miRNAs showed opposite trend. Among the miRNAs mentioned above, 18 miRNAs showed significant differentiation between plasma- and CSF-derived exosomal miRNAs (listed in Table 3).

Differentially expressed miRNAs of plasma-derived exosomes among different SCA3/MJD subtypes On the basis of the 69 upregulated miRNAs and 47 downregulated miRNAs detected from CSF-derived exosomes, these miRNA expression levels were further analyzed through the pairwise comparison among 1, 2 and 3 SCA3/MJD subtypes of their plasma-derived exosomes (Table 4).

\section{Differentially expressed miRNAs of CSF-derived exosomes among different SCA3/MJD subtypes}

Similar to above, the pairwise comparison of miRNAs expression in the CSF-derived exosomes among Type 1, 2 and 3 SCA3/MJD subtypes are listed in Table 4.

The differential expression of exosomal miRNAs with adjusted p-values mentioned above have been displayed in Supplementary Table 4.

Relative expression levels of miRNAs chosen for further verification in the validation cohort

After a verification by qPCR detection (primers are listed in Supplementary Table 3), the expression of mir-7014 was found to be significantly downregulated in patients' plasma-derived exosomes ( $\mathrm{p}<0.05$; Supplementary Table $1-2)$, which was in accordance with small RNA sequencing results. And after linear-regression analysis with clinical indexes, mir-7014 was found to be correlated with age at onset with a coefficient of 0.01493091 and an intercept of 26.890955696793025 (shown in Supplemental File 2). Besides, the trends of other miRNAs except mir-7014 were either unconspicuous or contrary to the small RNA sequencing results (Figure 1G). Moreover, Random Forest or LASSO seemed to be not that applicable for the present study, the evaluation results of which have been listed in the Supplementary File 4.

\section{miRNAs \& downstream target genes' prediction}

mir-7014 was predicted to be on Chromosome12 from 56908536 to 56908583 with a mature sequence 'cuguguuccugagccuug', a complementary sequence 'gucaguggcacagcc' and a precursor sequence 'gucaguggcacagccacugccugagggugcuguguuccugagccuug'. Characteristic hairpin structures of the detected miRNAs precursor were explored using MiRDeep2, and its structure predicted by mfold software (website: http://unafold.rna.albany.edu/?q=mfold) 


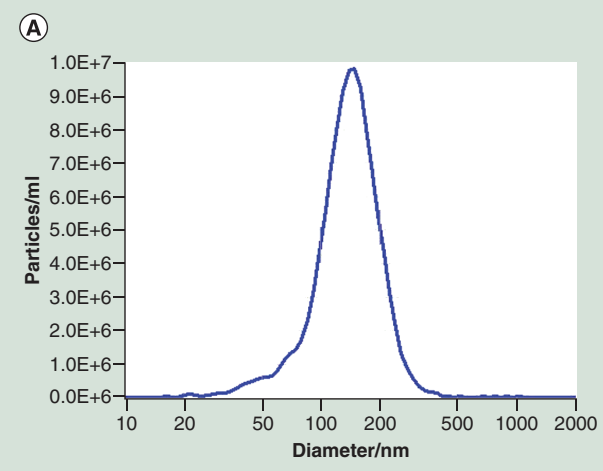

(D)

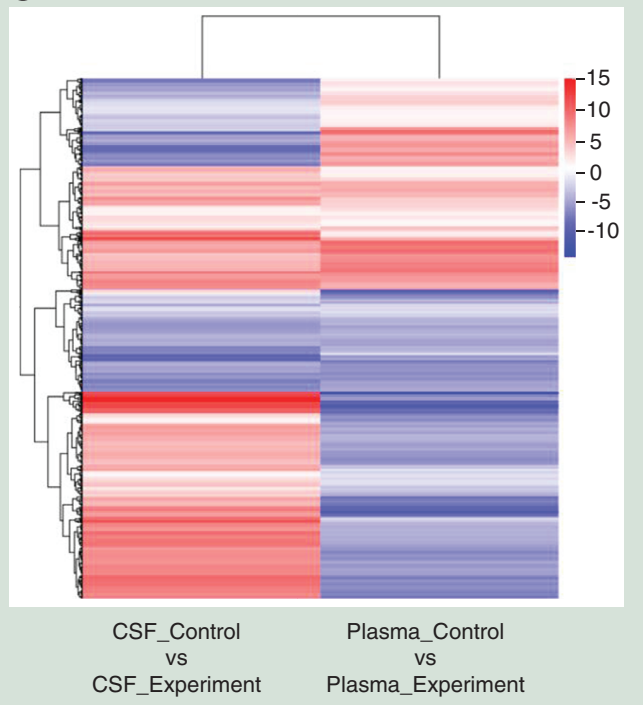

(B)

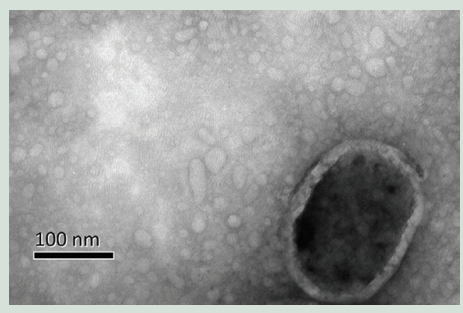

(C)

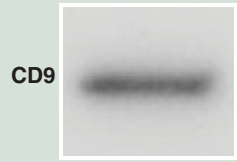

(E) TargetScan miRanda

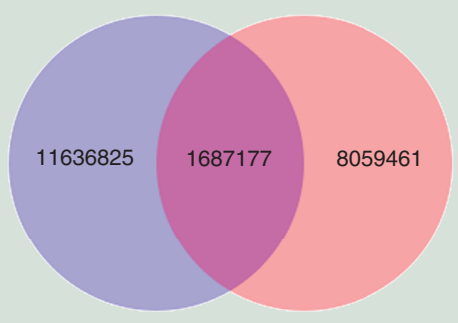

(F) TargetScan

miRanda

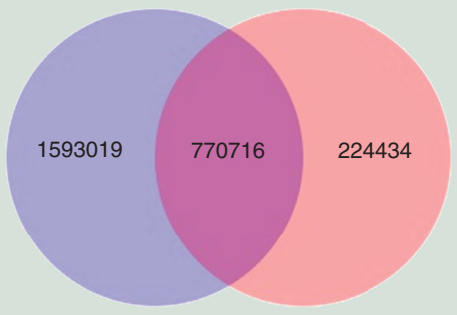

(G)

qPCR analysis

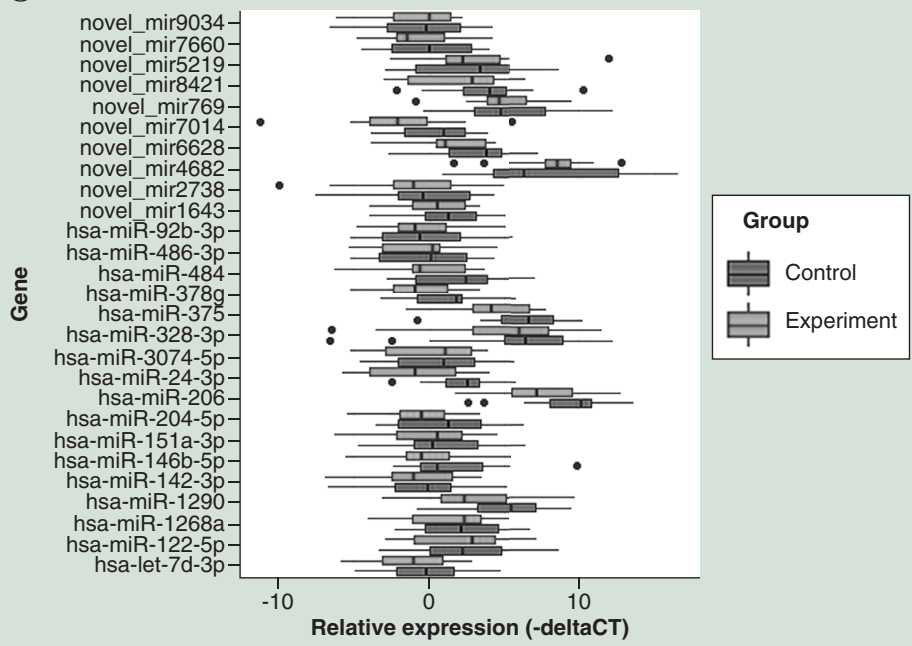

Figure 1. Screening and identification of differentially expressed exosomal miRNAs. (A) A representative depicting vesicle size and number of plasma- and CSF-derived exosomes from SCA3/MJD and control subjects. (B) A typical image of plasma- and CSF-derived exosomes screened by a transmission electron microscope. (C) Detection for expression of exosome marker CD9. (D) Hierarchical clustering among different comparing groups. The abscissa represents each comparing group and the ordinate means differentially expressed miRNAs. Colors indicate fold change (upregulated: red, downregulated: blue). (E) Venn statistics of the target predictors of the detected miRNAs. (F) Venn statistics of the filtered target predictors of the detected miRNAs. (G) Expression of miRNAs chosen for qPCR verification in the validation cohort. Gray box denotes relative expression of patients' plasma-derived exosomal miRNAs and dark gray box stands for that of the controls group.

CSF: Cerebrospinal fluid; SCA3/MJD: Spinocerebellar ataxia Type 3/Machado-Joseph disease; qPCR: Quantitative polymerase chain reaction. 
Table 2. Differential expression of miRNAs in plasma- and cerebrospinal fluid-derived exosomes.

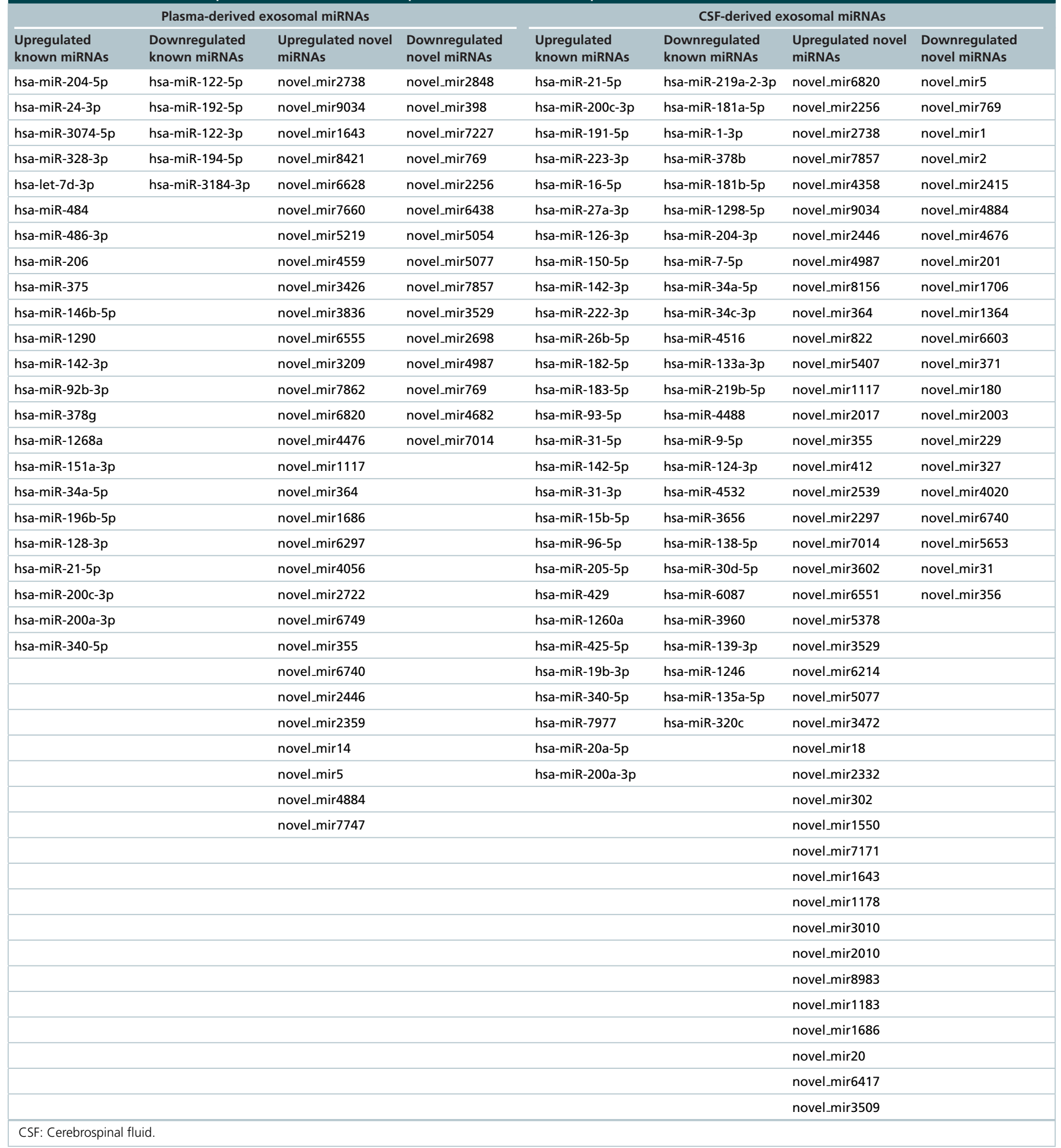

is displayed in Supplementary File 3. With regard to the target genes of all the detected miRNAs, 1687,177 common spots between TargetScan and RNA hybrid databases were identified as shown in Figure 1E. In order to minimize the possible interested downstream targets of these miRNAs, appropriate filter conditions including MFE and score evaluation were applied for the further analysis (Figure 1F). 
Table 3. Comparison between differentially expressed exosomal miRNAs derived from plasma and cerebrospinal fluid samples in spinocerebellar ataxia Type 3/Machado-Joseph disease patients.

\begin{tabular}{|c|c|c|c|c|c|}
\hline Plasma $\uparrow \operatorname{CSF} \uparrow$ & Plasma $\downarrow$ CSF $\downarrow$ & Plasma $\uparrow$ CSF $\downarrow$ & Plasma $\downarrow$ CSF $\uparrow$ & Plasma $/$ CSF $\uparrow$ & Plasma $/$ CSF $\downarrow$ \\
\hline hsa-miR-21-5p & novel_mir769 & hsa-miR-34a-5p & novel_mir2256 & hsa-miR-34a-5p & novel_mir2256 \\
\hline hsa-miR-200c-3p & & novel_mir5 & novel_mir7857 & novel_mir4884 & hsa-miR-200c-3p \\
\hline hsa-miR-142-3p & & novel_mir4884 & novel_mir4987 & novel_mir6740 & novel_mir2738 \\
\hline hsa-miR-340-5p & & novel_mir6740 & novel_mir7014 & & novel_mir7857 \\
\hline hsa-miR-200a-3p & & & novel_mir3529 & & novel_mir9034 \\
\hline novel_mir6820 & & & novel_mir5077 & & novel_mir2446 \\
\hline novel_mir2738 & & & & & novel_mir4987 \\
\hline novel_mir9034 & & & & & novel_mir364 \\
\hline novel_mir2446 & & & & & novel_mir1117 \\
\hline novel_mir364 & & & & & novel_mir355 \\
\hline novel_mir1117 & & & & & novel_mir7014 \\
\hline novel_mir355 & & & & & novel_mir3529 \\
\hline novel_mir1643 & & & & & novel_mir5077 \\
\hline \multirow[t]{2}{*}{ novel_mir1686 } & & & & & novel_mir1643 \\
\hline & & & & & novel_mir769 \\
\hline \multicolumn{6}{|c|}{$\begin{array}{l}\text { Plasma/CSF represents differentially expressed exosomal miRNAs with significant difference between plasma and CSF groups. } \\
\uparrow \text { stands for upregulated; } \downarrow \text { stands for downregulated exosomal miRNAs expression. } \\
\text { CSF: Cerebrospinal fluid. }\end{array}$} \\
\hline
\end{tabular}

GO enrichment \& KEGG pathway analysis of mir-7014

GO-based analysis was performed to further investigate the potential roles of mir-7014 involved in SCA3/MJD disease. A total of 4112 potential mir-7014 downstream targets were classified into biological processes, 3018 into cellular components and 1149 into molecular functions. The common biological processes terms were cellular process, single-organism process, metabolic process, biological regulation, regulation of biological process and response to stimulus. Some of the significant cellular components with the highest target numbers were cell, cell part, organelle and membrane. Binding and catalytic activity were among the most abundant classes in molecular function category (Figure 2A).

The KEGG classification system categorized the target genes of mir-7014 into different groups. The top 15 enriched groups among KEGG categories included signal transduction, overview of cancers, transport and catabolism, global and overview maps, immune system, folding sorting and degradation, cellular communityeukaryotes, endocrine system, translation, cancers: specific types, signal molecules and interaction, nervous system, infectious diseases (Figure 2B).

Moreover, KEGG pathway enrichment analysis of these target genes was carried out to identify potential pathways regulated by mir-7014 (Figure 2C). The results exhibited that target genes were enriched in protein processing in endoplasmic reticulum (Figure 3A) and axon guidance (Figure 3B).

\section{Discussion}

In view of all the results achieved from the present study, exosomal mir-7014 was found to be downregulated in SCA3/MJD patients' plasma, while upregulated in patients' CSF. Moreover, mir-7014 in CSF-derived exosomes from Type 1, 2 and 3 groups were successively upregulated with a statistical significance basing on the present study. Further, after analyzing the correlation of mir-7014 with clinical related indexes such as age at onset, SARA scores, CAG repeats and duration, we found that there was a correlation of it with age at onset, while no obvious correlation with other aspects in our present study, which may need more exploration in the future work. Consequently, exosomal mir-7014 is probably a significant biomarker candidate in SCA3/MJD, which indicates the clinical classification and age at onset. As one kind of important transport medium for noncoding RNAs, exosomes are more stable than their parent cells and could cross the blood-brain barrier, allowing communication between periphery and brain both in normal and disease conditions [48-50]. Exosomes could be released from all types of cells and bidirectionally transported through the blood-brain communication interfaces, blood-brain and blood-cerebrospinal fluid barriers [51]. As to our results, there is an opposite trend between plasma- and CSF-derived exosomal mir-7014 expression levels, how this trend formed deserves to be discussed. According to 


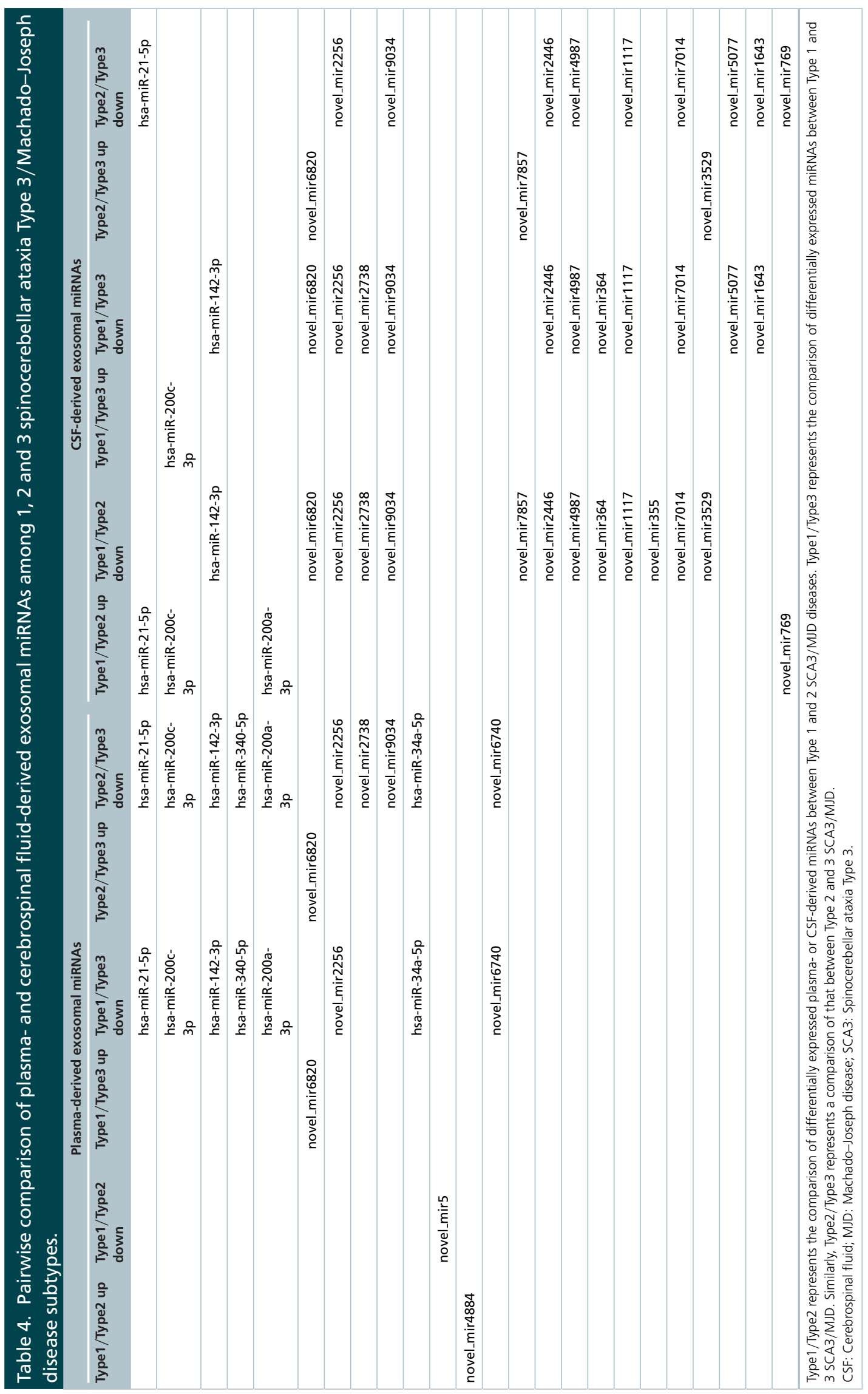




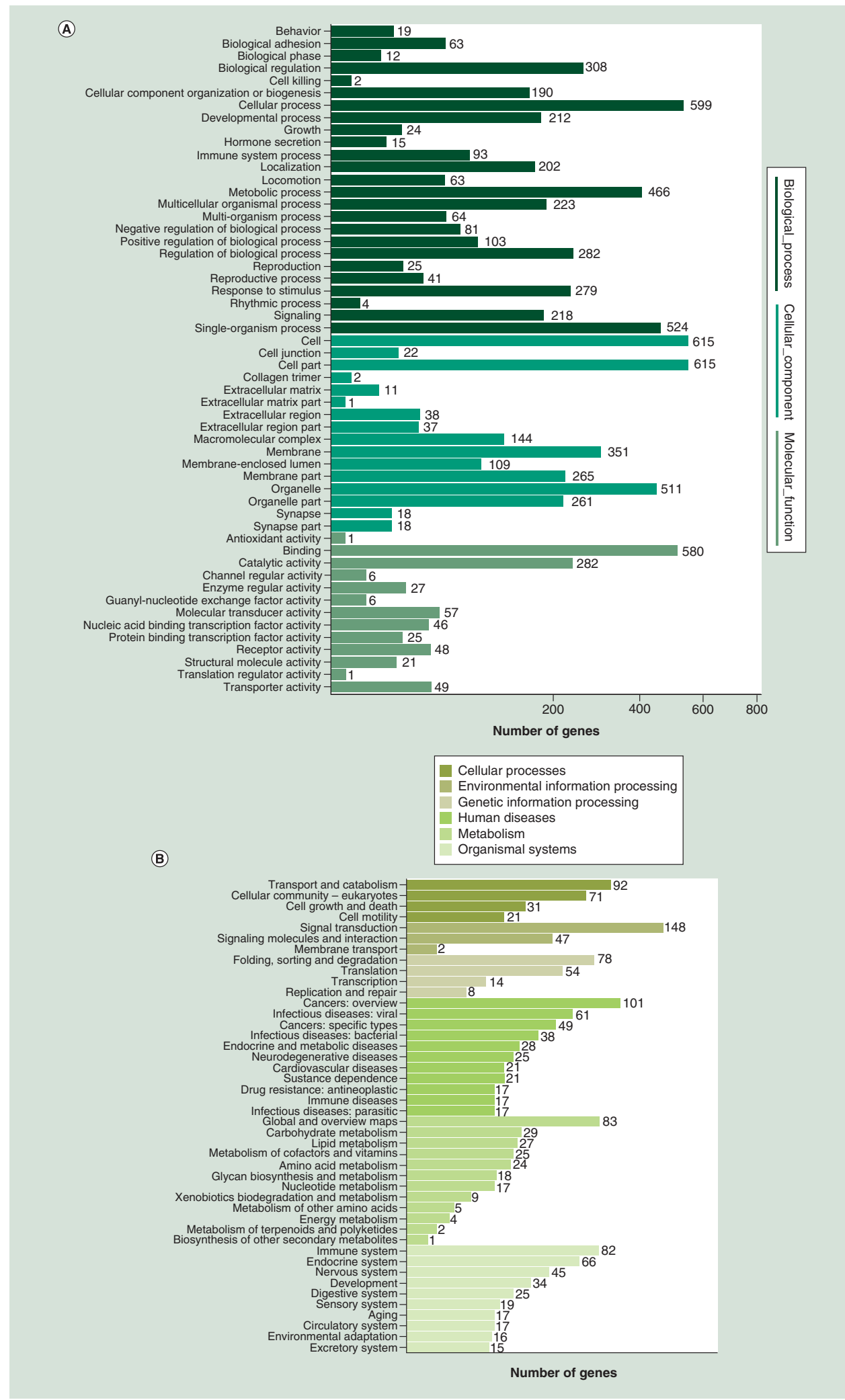

Figure 2. GO and KEGG analyses of target genes and downstream pathways of mir-7014. (A) GO annotation of downstream target genes of mir-7014. The abscissa is the target gene number and the ordinate left is the GO annotation of mir-7014. (B) KEGG classification of the target genes of mir-7014. The abscissa is the target gene number and the ordinate left is the KEGG classification of mir-7014.

(C) KEGG pathway enrichment analysis of the target genes of mir-7014. The abscissa is the rich factor and the ordinate left is the pathway classification of mir-7014. Rich factor represents the frequency of differentially expressed genes with pathway annotation in all the genes with annotation. The size of dots indicates gene number for enrichment and the color shade of them prompt the Q value. The smaller the $Q$ value is, the better the enrichment evaluation is.

GO: Gene ontology; KEGG: Kyoto Encyclopedia of Genes and Genomes. 


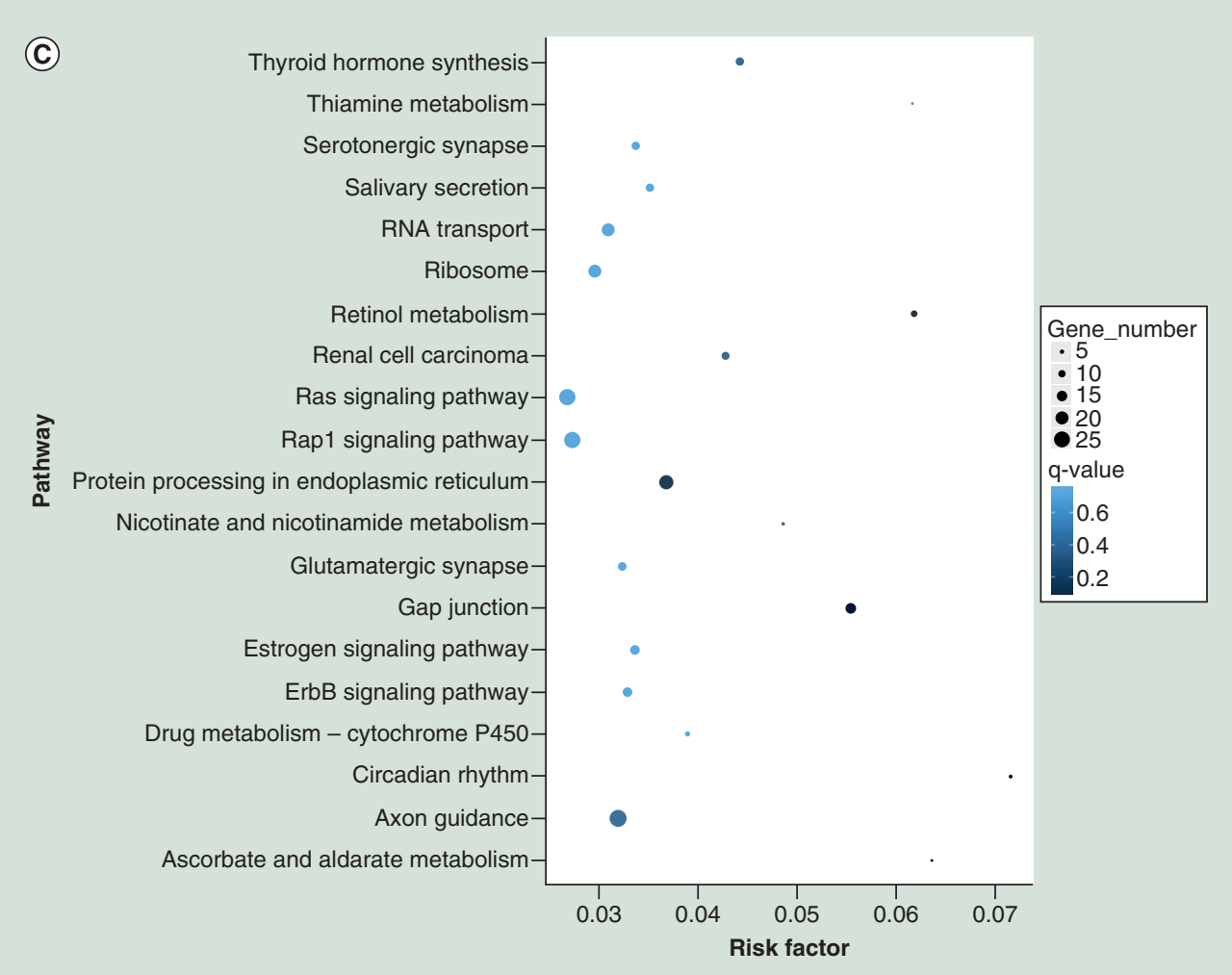

Figure 2. GO and KEGG analyses of target genes and downstream pathways of mir-7014 (cont.). (A) GO annotation of downstream target genes of mir-7014. The abscissa is the target gene number and the ordinate left is the GO annotation of mir-7014. (B) KEGG classification of the target genes of mir-7014. The abscissa is the target gene number and the ordinate left is the KEGG classification of mir-7014. (C) KEGG pathway enrichment analysis of the target genes of mir-7014. The abscissa is the rich factor and the ordinate left is the pathway classification of mir-7014. Rich factor represents the frequency of differentially expressed genes with pathway annotation in all the genes with annotation. The size of dots indicates gene number for enrichment and the color shade of them prompt the $Q$ value. The smaller the $Q$ value is, the better the enrichment evaluation is.

GO: Gene ontology; KEGG: Kyoto Encyclopedia of Genes and Genomes.

the communication between CSF and plasma, some interflow between them may occur, for example, what if plasma-derived exosomal mir-7014 could be transferred from peripheral blood into CSF through blood-brain barrier? All the above assumption makes it interesting for us to carry out further exploration.

To better understand the functions of novel mir-7014, all suspected downstream genes with differential expression levels were classified into different groups according to their function annotation and pathways enrichment. It is of interest that KEGG pathway annotation of target genes resulted in the identification of two highly enriched pathways in our results: protein processing in endoplasmic reticulum and axon guidance, both of which have been reported to be involved in the pathogenesis of SCA3/MJD [52-54]. Endoplasmic reticulum-associated degradation is the system that mediates the ubiquitination of misfolded proteins or unassembled complex constituents present in the secretory pathway and their exportation to the cytosol for degradation by the proteasome $[12,13,55-57]$. In the pathway of protein processing in endoplasmic reticulum (Figure 3A), protein processing is divided into two pathways: the correctly folded protein would be transported into the Golgi body via the ERManI-mediated pathway and released into the cytoplasm; while the misfolded protein would be degraded through EDEM and ERManI, in which the family of heat shock proteins such as HSP40/HSP70 could participate. While ataxin 3 had been documented to be associated with the endoplasmic reticulum-associated degradation, there were different points of view whether ataxin 3 promoted or decreased degradation through this pathway [13,58]. A modeling study by Brown et al. [52] demonstrated that IP3-mediated calcium release in the Purkinje neuron could activate voltage-gated KCa channels, namely BK and IK, and provided insight into the interplay between smooth endoplasmic reticulum calcium channel of IP3R1 sensitivity and abundance in the function and dysfunction of the Purkinje cell. Reina 


\section{(A) Protein processing in endoplasmic reticulum}

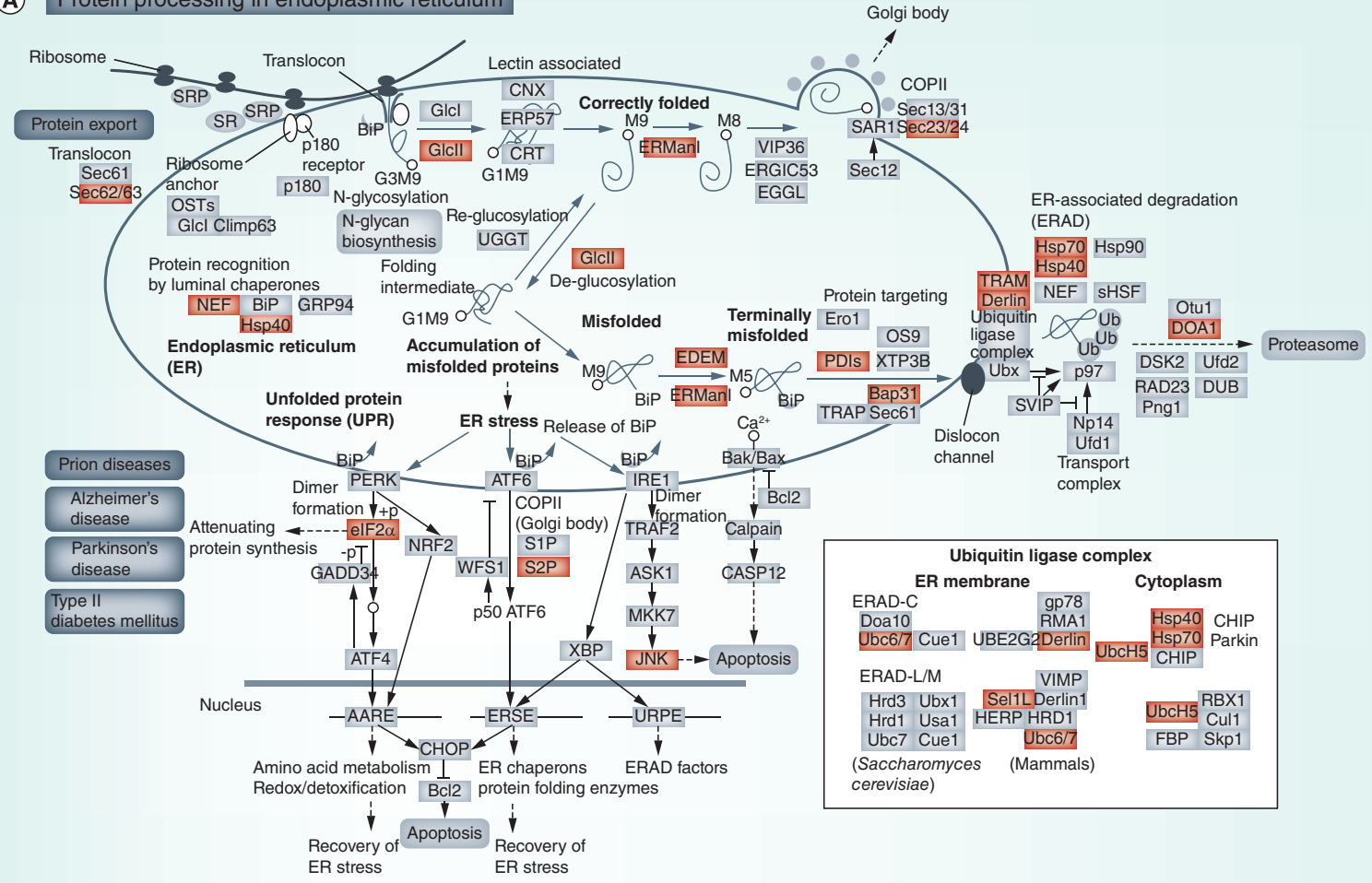

(B)

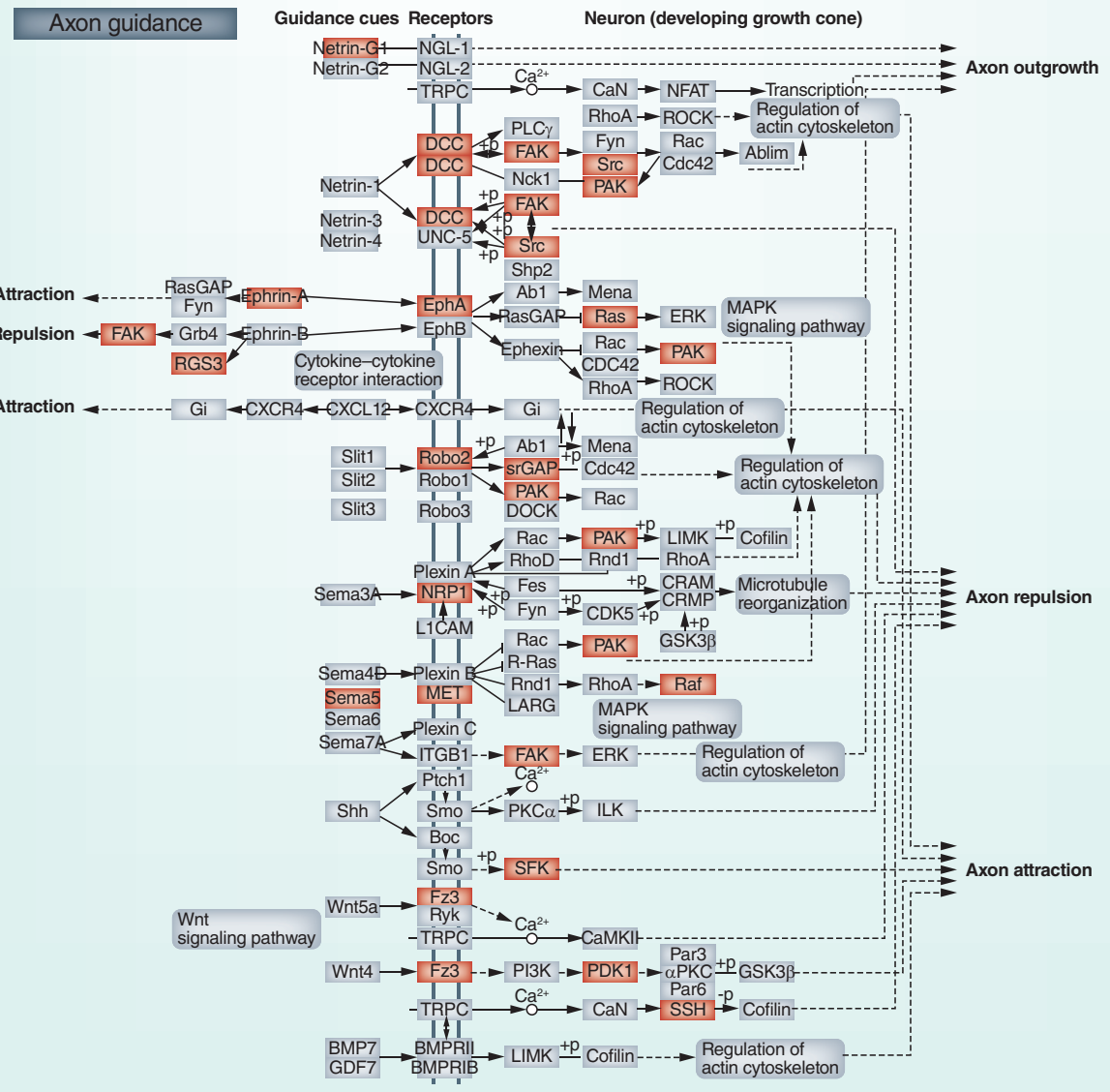

Figure 3. KEGG pathway maps for protein processing in endoplastic reticulum and axon guidance pathways. (A) KEGG pathway map for protein processing in endoplasmic reticulum pathway. The red boxes indicate the potential target genes of mir-7014 in this particular pathway. (B) KEGG pathway map for axon guidance pathway. The red boxes indicate the potential target genes of mir-7014 in this particular pathway. 
et al. [53] pointed out that ER stress could increase misfolded and aberrant proteins without inducing nuclear accumulation of ataxin3, which might reflect a primarily cytoplasmic function for ataxin3 following proteotoxic stresses. In the axon guidance pathway (Figure 3B), genes such as PAK, SEMA5 and EPHA play important roles in physiological processes such as axon outgrowth and repulsion. There also have been other SCA3 studies related with this pathway, for example, transcriptional changes in brain tissue of the SCA3/MJD mice were observed by Toonen et al. [54], most transcriptional changes occurred in brainstem and striatum, while cerebellum and cortex were only modestly affected. When examining individual brain regions, axon guidance and synaptic transmission pathway were most strongly altered in striatum. All the above threw light on many interesting clues for us, which may have an important potential for further exploration.

Combining the pathway annotations and all the above findings with the previous studies, mir-7014 could probably be a significant regulator of some SCA3/MJD-related genes involved in the pathogenesis process of SCA3/MJD. Moreover, if the exosomal mir-7014 is transferred from peripheral vessels to CNS, exosomes could be served as a potential therapeutic carrier for SCA3/MJD. The exosomes encapsulated with mir-7014 may be delivered from the patients' peripheral vessels to CSF so as to prevent the disease progression. As a result, further studies about the biological functions and the regulation mechanisms of mir-7014 will provide us with more prospective application of exosomal miRNAs in SCA3/MJD diagnosis and treatment.

Up to now, there is not a complete system related to exosomes research in neurodegenerative diseases formed yet, the exploration about how exosomal miRNAs function is not profound enough, and even without any relevant reports in SCAs. However, several studies about the roles of free miRNAs in SCAs have existed: first of all, in our former study, we found that miR-25, miR-125b, miR-29a and miR-34b could be potential biomarkers for SCA3/MJD. Intersected with that, miR-29a was also found to be downregulated in a cellular model of SCA17 [59]. Further, there also have been some miRNA studies in cell models or animal models from SCA3/MJD: in human neuronal cultures derived from SCA3 patient-specific induced pluripotent stem cell (iPSC) lines, miR-370 and miR-543 levels were found to be upregulated, while DNAJB1 expression was concurrently reduced. Based on these findings, Evert et al. [60] proposed that downregulation of DNAJB1 by miR-370 and miR-543 could be an early event contributing to SCA3 pathogenesis, therefore, inhibition of these two miRNAs in turn could stabilize DNAJB1 and thereby be beneficial in SCA3 disease. Three other miRNAs, mir-9, mir-181a, and mir-494, have been reported to interact with the ATXN3-3' UTR, whose expression was found to be dysregulated in human MJD neurons and in other MJD cell and animal models. Overexpression of these miRNAs in mice could reduce mut-ATXN3 levels, aggregate counts, and neuronal dysfunction [61]. Bilen et al. [62] put forward that miRNA pathways could modulate poly-Q-induced neurodegeneration in Drosophila and human cells. Some also carried out research in SCA3-lymphoblastoid cells and proposed that miR-25 and miR-181 family members could bind to the 3'UTR cooperatively to suppress the expression of ATXN3. Besides, McCann et al. [63] found that in the Drosophila model of SCA2, a direct homolog of the disease-associated protein Atx2 interacted with the important effector protein ago 1 of miRNAs, whereas the latter was responsible for the effectiveness of miRNAs-mediated gene translation inhibition. However, the interaction of Atx2-ago1 has not been verified in mouse or human models, and the role of the extended mutant ataxin-2 protein needs to be further elucidated.

Above all, there are a number of similar designed studies using exosomal miRNAs as biomarkers in other neurodegenerative diseases: Yang et al. [64] found that both serum exosomal miR-135a and miR-384 were upregulated while miR-193b was downregulated in serum of AD patients compared with that of normal controls; Furthermore, they declared that exosome miR-384 was the best among the three miRNAs to discriminate $\mathrm{AD}, \mathrm{VaD}$ and PDD, and the combination of miR-135a, -193b and -384 was better than a particular one for early AD diagnosis. Some [65] other researchers developed a miRNA profiling strategy for exosomal miRNAs isolated from cerebrospinal fluid (CSF) in PD and AD and found that 16 exosomal miRNAs were upregulated and 11 miRNAs were downregulated significantly in PD-CSF when compared with those in healthy controls (relative fold $>2, \mathrm{p}<0.05$ ). MiR-1 and miR-19b-3p were significantly reduced, while miR-153, miR-409-3p, miR-10a-5p and let-7g-3p were significantly overexpressed in PD-CSF exosomes. Further bioinformatic analysis by DIANA-mirPath demonstrated that neurotrophin signaling, mTOR signaling, ubiquitin mediated proteolysis, dopaminergic synapse and glutamatergic synapse were the most prominent pathways enriched in quantiles with PD miRNA patterns; Moreover, after analyzing a panel of 760 miRNAs in the CSF of ten $\mathrm{AD}$ patients and ten healthy subjects and validation in a larger group by individual qPCR assays, some scholars proposed miR-9-5p and miR-598 as potential biomarkers for AD.

More interestingly, we found that in animal models of HD, which had similar pathogenesis with SCA3/MJD, some researchers [60] ever developed an exosome-based delivery method to treat this $\mathrm{HD}$ as follows: exosomes 
were harvested from a stable cell line stably overexpressed with miR-124, which exhibited a high level of miR-124 expression. After injecting the above Exo-miR-124 into the striatum of R6/2 transgenic HD mice, expression of the target gene, RE1-Silencing Transcription Factor, was reduced. As we can see, all the above explorations prove that exosomal miRNAs will have a tremendous potential in the field of neurodegenerative disease, in the meantime, more and more scholars are devoting their effort in this field.

On the other hand, with respect to the other miRNAs except mir-7014 that we chose for qPCR verification, we found that the trends of miRNAs expression levels in plasma-derived exosomes were not in consistent with that obtained from the small RNA sequencing (either without significant difference or being opposite to that). One rational reason to explain this interesting discrepancy may be the scale of the collected sample size not large enough for an integrated illustration. Besides, some analysis deviation may be generated due to platform differences for verification. Moreover, some other differentially expressed miRNAs obtained from small RNA sequencing results without our verification remain to be further investigated in SCA3/MJD disease.

It is summarized that exosomal mir-7014 may be a potential biomarker of SCA3/MJD disease suggesting the clinical classification and age at onset of SCA3/MJD. Above all, this is the first research of exosomal miRNAs in SCAs internationally.

\title{
Future perspective
}

For all this, there are still some limitations or deficiency to be improved in our future work over the next few years. For instance, as it is really hard, at least in this study, to control the sample collecting time strictly, the variance of mir-7014 expression measures at different times could hardly be avoided. With respect to exosomes, more detailed indexes such as the polydispersity index of exosomes could not be covered by ZetaView Nanoparticles Trace Analyzer, which will be improved in our future exosome research. Moreover, as a preliminary study, further tests about the functional regulation of exosomal mir-7014 in cell models and mouse models are necessary, which would have important value for preclinical studies of therapeutic intervention.

\section{Summary points}

- Exosomes in spinocerebellar ataxia Type 3 (SCAs) were explored for the first time internationally.

- Both plasma and cerebrospinal fluid (CSF) were chosen for detection in this study.

- Apart from comparing exosomal miRNAs between SCA3/Machado-Joseph disease (MJD) patients and controls, their expression levels in three subtypes of SCA3/MJD were also intercompared.

- Total 53 miRNAs (30 novel miRNAs and 23 known miRNAs) were upregulated and 20 miRNAs (15 novel miRNAs and five known miRNAs) were downregulated significantly in exosomes derived from SCA3/MJD patients' plasma compared with that from SCA3/MJD-free controls.

- Total 69 miRNAs (41 novel miRNAs and 28 known miRNAs) were upregulated and 47 miRNAs (21 novel miRNAs and 26 known miRNAs) were downregulated significantly in exosomes derived from SCA3/MJD patients' CSF compared with that from SCA3/MJD-free controls.

- One novel miRNA of mir-7014 is notably downregulated in plasma-derived exosomes, while upregulated in CSF-derived exosomes of SCA3 patients.

- Mir-7014 is successively upregulated in CSF-derived exosomes from Type 1, Type 2 and Type 3 groups.

- Two highly enriched pathways among mir-7014's target genes, which are probably involved in the pathogenesis of SCA3/MJD, were identified in this study: protein processing in endoplasmic reticulum and axon guidance.

\author{
Supplementary data \\ To view the supplementary data that accompany this paper please visit the journal website at: \\ www.futuremedicine.com/doi/full/10.2217/epi-2019-0081
}

Author contributions

$X$ Hou, L Zhang and $\mathrm{H}$ Jiang conceived and designed the experiments. X Hou, X Gong, L Zhang, T Li and $Y$ Xie collected the samples and performed the experiments. X Hou and L Zhang analyzed the data. H Yuan, Y Peng, R Qiu, K Xia, B Tang and H Jiang contributed equipment, materials and analysis tools. 


\section{Acknowledgments}

We are indebted to the SCA3/MJD patient and control individuals who have participated in this study. We are grateful to Prof. Hong Shen for helpful suggestions.

Financial \& competing interests disclosure

This work was supported by the National KeyResearch and Development Program of China (grant numbers 2016 YFC0905100 and 2016 YFC0901504 to H Jiang), the National Natural Science Foundation of China (grant number 81771231 to H Jiang, grant number 81600995 to Y Shi), Scientific Research Foundation of Health Commission of Hunan Province (grant number B2019183 to $\mathrm{H}$ Jiang) the Key Research and Development Program of Hunan Province (grant number 2018SK2092 to H Jiang), the Clinical and rehabilitation fund of Peking University Weiming Biotech Group (grant number xywm2015/10 to H Jiang). The National Natural Science Foundation of China (grant number 81671123 to L Zhang). No authors have received any funding from any institution, including personal relationships, interests, grants, employment, affiliations, patents, inventions, honoraria, consultancies, royalties, stock options/ownership or expert testimony for the last 36 months. The authors have no other relevant affiliations or financial involvement with any organization or entity with a financial interest in or financial conflict with the subject matter or materials discussed in the manuscript apart from those disclosed.

No writing assistance was utilized in the production of this manuscript.

Ethical conduct of research

This research was approved by Medical Ethics Committee of Xiangya Hospital, Central South University in China, which was in accordance with the ethical standards in the Declaration of Helsinki. In addition, for investigations involving human subjects, informed consent has been obtained from the participants involved.

Data sharing statement

The data and materials generated during the study are available from the corresponding author on reasonable request. The datasets generated during the current study are available at the Sequence Read Archive (SRA) repository under accession code PRJNA522863. Confidential patient data have not been shared.

\section{Open access}

This work is licensed under the Attribution-NonCommercial-NoDerivatives 4.0 Unported License. To view a copy of this license, visit http://creativecommons.org/licenses/by-nc-nd/4.0/

\section{References}

Papers of special note have been highlighted as: $\bullet$ of interest; $\bullet \bullet$ of considerable interest

1. Paulson H. Machado-Joseph disease/spinocerebellar ataxia type 3. Handb. Clin. Neurol. 103, 437-449 (2012).

2. Costa Mdo C, Paulson HL. Toward understanding Machado-Joseph disease. Prog. Neurobiol. 97(2), 239-257 (2012).

- A review of spinocerebellar ataxia Type 3/Machado-Joseph disease (SCA3/MJD) focusing primarily on the function and dysfunction of ATXN3 and on advances toward potential therapies.

3. Chen $\mathrm{Z}$, Zheng $\mathrm{C}$, Long $\mathrm{Z}$ et al. (CAG)n loci as genetic modifiers of age-at-onset in patients with Machado-Joseph disease from mainland China. Brain 139(Pt 8), e41 (2016).

- Comments on modulation of the age at onset in spinocerebellar ataxia by CAG tracts in various genes.

4. Maciel P, Costa MC, Ferro A et al. Improvement in the molecular diagnosis of Machado-Joseph disease. Arch. Neurol. 58(11), 1821-1827 (2001).

5. Long Z, Chen Z, Wang C et al. Two novel SNPs in ATXN3 $3^{\prime}$ UTR may decrease age at onset of SCA3/MJD in Chinese patients. PLoS ONE 10(2), e0117488 (2015).

6. Bettencourt C, Lima M. Machado-Joseph disease: from first descriptions to new perspectives. Orphanet J. Rare Dis. 6, 35 (2011).

7. Kawaguchi Y, Okamoto T, Taniwaki M et al. CAG expansions in a novel gene for Machado-Joseph disease at chromosome 14q32.1. Nat. Genet. 8(3), 221-228 (1994).

8. Takiyama Y, Oyanagi S, Kawashima S et al. A clinical and pathologic study of a large Japanese family with Machado-Joseph disease tightly linked to the DNA markers on chromosome 14q. Neurology 44(7), 1302-1308 (1994).

9. Li F, Macfarlan T, Pittman RN, Chakravarti D. Ataxin-3 is a histone-binding protein with two independent transcriptional corepressor activities. J. Biol. Chem. 277(47), 45004-45012 (2002).

10. Evert BO, Araujo J, Vieira-Saecker AM et al. Ataxin-3 represses transcription via chromatin binding, interaction with histone deacetylase 3, and histone deacetylation. J. Neurosci. 26(44), 11474-11486 (2006).

11. Chou AH, Yeh TH, Ouyang P, Chen YL, Chen SY, Wang HL. Polyglutamine-expanded ataxin-3 causes cerebellar dysfunction of SCA3 transgenic mice by inducing transcriptional dysregulation. Neurobiol. Dis. 31(1), 89-101 (2008). 
12. Burnett B, Li F, Pittman RN. The polyglutamine neurodegenerative protein ataxin-3 binds polyubiquitylated proteins and has ubiquitin protease activity. Hum. Mol. Gen. 12(23), 3195-3205 (2003).

13. Wang Q, Li L, Ye Y. Regulation of retrotranslocation by p97-associated deubiquitinating enzyme ataxin-3. J. Cell Biol. 174(7), 963-971 (2006).

14. Wang C, Chen Z, Peng H et al. Investigation on modulation of DNA repair pathways in Chinese MJD patients. Neurobiol. Aging 71, 267.e265-267.e266 (2018).

\section{- Epigenetic research on SCA3/MJD in our former study.}

15. Paulson HL, Perez MK, Trottier Y et al. Intranuclear inclusions of expanded polyglutamine protein in spinocerebellar ataxia type 3 . Neuron 19(2), 333-344 (1997).

16. Perez MK, Paulson HL, Pendse SJ, Saionz SJ, Bonini NM, Pittman RN. Recruitment and the role of nuclear localization in polyglutamine-mediated aggregation. J. Cell Biol. 143(6), 1457-1470 (1998).

17. Chai Y, Koppenhafer SL, Shoesmith SJ, Perez MK, Paulson HL. Evidence for proteasome involvement in polyglutamine disease: localization to nuclear inclusions in SCA3/MJD and suppression of polyglutamine aggregation in vitro. Hum. Mol. Gen. 8(4), 673-682 (1999).

18. Fujigasaki H, Uchihara $\mathrm{T}$, Koyano $\mathrm{S}$ et al. Ataxin-3 is translocated into the nucleus for the formation of intranuclear inclusions in normal and Machado-Joseph disease brains. Exp. Neurol. 165(2), 248-256 (2000).

19. Nucifora FC Jr., Ellerby LM, Wellington CL et al. Nuclear localization of a non-caspase truncation product of atrophin-1, with an expanded polyglutamine repeat, increases cellular toxicity. J. Biol. Chem. 278(15), 13047-13055 (2003).

20. Kretzschmar D, Tschape J, Bettencourt Da Cruz A et al. Glial and neuronal expression of polyglutamine proteins induce behavioral changes and aggregate formation in Drosophila. Glia 49(1), 59-72 (2005).

21. Klement IA, Skinner PJ, Kaytor MD et al. Ataxin-1 nuclear localization and aggregation: role in polyglutamine-induced disease in SCA1 transgenic mice. Cell 95(1), 41-53 (1998).

22. Bichelmeier $\mathrm{U}, \mathrm{Schmidt} \mathrm{T}$, Hubener $\mathrm{J}$ et al. Nuclear localization of ataxin-3 is required for the manifestation of symptoms in SCA3: in vivo evidence. J. Neurosci. 27(28), 7418-7428 (2007).

23. Long Z, Li T, Chen Z et al. Cerebellar lncRNA expression profile analysis of SCA3/MJD mice. Int. J. Genomics 2018, 5383517 (2018).

- Research on noncoding RNAs in SCA3/MJD in our former study.

24. Tkach M, Thery C. Communication by extracellular vesicles: where we are and where we need to go. Cell 164(6), 1226-1232 (2016).

25. Shi Y, Wang C, Huang F et al. High serum GFAP levels in SCA3/MJD may not correlate with disease progression. Cerebellum 14(6), 677-681 (2015).

26. Saman S, Kim W, Raya M et al. Exosome-associated tau is secreted in tauopathy models and is selectively phosphorylated in cerebrospinal fluid in early Alzheimer disease. J. Biol. Chem. 287(6), 3842-3849 (2012).

27. Mullins RJ, Mustapic M, Goetzl EJ, Kapogiannis D. Exosomal biomarkers of brain insulin resistance associated with regional atrophy in Alzheimer's disease. Human Brain Mapp. 38(4), 1933-1940 (2017).

28. Stuendl A, Kunadt M, Kruse N et al. Induction of alpha-synuclein aggregate formation by CSF exosomes from patients with Parkinson's disease and dementia with Lewy bodies. Brain 139(Pt 2), 481-494 (2016).

29. Jeon I, Cicchetti F, Cisbani G et al. Human-to-mouse prion-like propagation of mutant huntingtin protein. Acta Neuropathol. 132(4), 577-592 (2016).

30. Vader P, Mol EA, Pasterkamp G, Schiffelers RM. Extracellular vesicles for drug delivery. Adv. Drug Deliv. Rev. 106(Pt A), 148-156 (2016).

31. Cogswell JP, Ward J, Taylor IA et al. Identification of miRNA changes in Alzheimer's disease brain and CSF yields putative biomarkers and insights into disease pathways. J. Alzheimer Dis. 14(1), 27-41 (2008).

\section{- Identification of miRNAs as biomarkers in the cerebrospinal fluid from neurodegenerative disease.}

32. Wang WX, Huang Q, Hu Y, Stromberg AJ, Nelson PT. Patterns of microRNA expression in normal and early Alzheimer's disease human temporal cortex: white matter versus gray matter. Acta Neuropathol. 121(2), 193-205 (2011).

33. Huang F, Zhang L, Long Z et al. miR-25 alleviates polyQ-mediated cytotoxicity by silencing ATXN3. FEBS Lett. 588(24), 4791-4798 (2014).

34. Gallo A, Tandon M, Alevizos I, Illei GG. The majority of microRNAs detectable in serum and saliva is concentrated in exosomes. PLoS ONE 7(3), e30679 (2012).

35. Monopoli MP, Zendrini A, Wu D et al. Endogenous exosome labelling with an amphiphilic NIR-fluorescent probe. Chem. Comm. 54(52), 7219-7222 (2018).

36. Mazzeo C, Canas JA, Zafra MP et al. Exosome secretion by eosinophils: a possible role in asthma pathogenesis. J. Allergy Clin. Immunol. 135(6), 1603-1613 (2015).

37. Langmead B, Trapnell C, Pop M, Salzberg SL. Ultrafast and memory-efficient alignment of short DNA sequences to the human genome. Genome Biol. 10(3), R25 (2009). 
38. Friedlander MR, Chen W, Adamidi C et al. Discovering microRNAs from deep sequencing data using miRDeep. Nat. Biotechnol. 26(4), 407-415 (2008).

39. Agarwal V, Bell GW, Nam JW, Bartel DP. Predicting effective microRNA target sites in mammalian mRNAs. eLife 4 (2015).

40. John B, Enright AJ, Aravin A, Tuschl T, Sander C, Marks DS. Human microRNA targets. PLoS Biol. 2(11), e363 (2004).

41. Jiang H, Wong WH. Statistical inferences for isoform expression in RNA-Seq. Bioinformatics 25(8), 1026-1032 (2009).

42. Wang L, Feng Z, Wang X, Wang X, Zhang X. DEGseq: an R package for identifying differentially expressed genes from RNA-seq data. Bioinformatics 26(1), 136-138 (2010).

43. Yang YH, Dudoit S, Luu P et al. Normalization for cDNA microarray data: a robust composite method addressing single and multiple slide systematic variation. Nucleic Acids Res. 30(4), e15 (2002).

44. Storey JD, Tibshirani R. Statistical significance for genomewide studies. Proc. Natl Acad. Sci. USA 100(16), 9440-9445 (2003).

45. Benjamini Y, Yosef H. Controlling the false discovery rate: a practical and powerful approach to multiple testing. J. R. Stat. Soc. Series B. Stat. Methodol. 57(1), 289-300 (1995).

46. Armstrong RA. When to use the Bonferroni correction. Ophthalmic Physiol. Opt. 34(5), 502-508 (2014).

47. Kanehisa M, Araki M, Goto S et al. KEGG for linking genomes to life and the environment. Nucleic Acids Res. 36(Database issue), D480-484 (2008).

48. Xiong Y, Mahmood A, Chopp M. Emerging potential of exosomes for treatment of traumatic brain injury. Neural Regen. Res. 12(1), 19-22 (2017).

49. Saeedi S, Israel S, Nagy C, Turecki G. The emerging role of exosomes in mental disorders. Transl. Psychiatry. 9(1), 122 (2019).

50. Zagrean AM, Hermann DM, Opris I, Zagrean L, Popa-Wagner A. Multicellular crosstalk between exosomes and the neurovascular unit after cerebral ischemia. therapeutic implications. Front. Neurosci. 12, 811 (2018).

51. Balusu S, Van Wonterghem E, De Rycke R et al. Identification of a novel mechanism of blood-brain communication during peripheral inflammation via choroid plexus-derived extracellular vesicles. EMBO Mol. Med. 8(10), 1162-1183 (2016).

52. Brown SA, Loew LM. Computational analysis of calcium signaling and membrane electrophysiology in cerebellar Purkinje neurons associated with ataxia. BMC Syst. Biol. 6, 70 (2012).

-• Protein processing in endoplasmic reticulum pathway reported to be involved in the pathogenesis of SCA3/MJD.

53. Reina CP, Zhong X, Pittman RN. Proteotoxic stress increases nuclear localization of ataxin-3. Hum. Mol. Gen. 19(2), 235-249 (2010).

-• Protein processing in endoplasmic reticulum pathway reported to be involved in the pathogenesis of SCA3/MJD.

54. Toonen LJA, Overzier M, Evers MM et al. Transcriptional profiling and biomarker identification reveal tissue specific effects of expanded ataxin-3 in a spinocerebellar ataxia type 3 mouse model. Mol. Neurodegen. 13(1), 31 (2018).

-• Axon guidance pathway reported to be involved in the pathogenesis of SCA3/MJD.

55. Albrecht M, Golatta M, Wullner U, Lengauer T. Structural and functional analysis of ataxin-2 and ataxin-3. Eur. J. Biochem. 271(15), 3155-3170 (2004).

56. Doss-Pepe EW, Stenroos ES, Johnson WG, Madura K. Ataxin-3 interactions with rad23 and valosin-containing protein and its associations with ubiquitin chains and the proteasome are consistent with a role in ubiquitin-mediated proteolysis. Mol. Cell. Biol. 23(18), 6469-6483 (2003).

57. Wang Q, Song C, Li CC. Molecular perspectives on p97-VCP: progress in understanding its structure and diverse biological functions. J. Struct. Biol. 146(1-2), 44-57 (2004).

58. Zhong X, Pittman RN. Ataxin-3 binds VCP/p97 and regulates retrotranslocation of ERAD substrates. Human Mol. Genet. 15(16), 2409-2420 (2006).

59. Roshan R, Ghosh T, Gadgil M, Pillai B. Regulation of BACE1 by miR-29a/b in a cellular model of Spinocerebellar Ataxia 17. RNA Biol. 9(6), 891-899 (2012).

60. Evert BO, Nalavade R, Jungverdorben J et al. Upregulation of miR-370 and miR-543 is associated with reduced expression of heat shock protein 40 in spinocerebellar ataxia type 3. PLoS ONE 13(8), e0201794 (2018).

61. Carmona V, Cunha-Santos J, Onofre I et al. Unravelling endogenous microRNA system dysfunction as a new pathophysiological mechanism in Machado-Joseph disease. Mol. Ther. 25(4), 1038-1055 (2017).

62. Bilen J, Liu N, Burnett BG, Pittman RN, Bonini NM. MicroRNA pathways modulate polyglutamine-induced neurodegeneration. Mol. Cell 24(1), 157-163 (2006).

63. Mccann C, Holohan EE, Das $\mathrm{S}$ et al. The Ataxin-2 protein is required for microRNA function and synapse-specific long-term olfactory habituation. Proc. Natl Acad. Sci. USA 108(36), E655-662 (2011).

64. Yang TT, Liu CG, Gao SC, Zhang Y, Wang PC. The serum exosome derived microRNA-135a, -193b, and -384 were potential Alzheimer's disease biomarkers. Biomed. Environ. Sci. 31(2), 87-96 (2018).

65. Gui Y, Liu H, Zhang L, Lv W, Hu X. Altered microRNA profiles in cerebrospinal fluid exosome in Parkinson disease and Alzheimer disease. Oncotarget 6(35), 37043-37053 (2015). 